\title{
La femme mal barrée. Croyances en La femme, de l'hystérie aux théories psychanalytiques
}

\author{
Fanny Chevalier*!
}

La question "qu'est-ce qu'une femme?" et la logique de l'exception sur laquelle se règle l'hystérique dans son rapport à la féminité témoignent de sa croyance selon laquelle la barre portée par Lacan sur La femme n'existe pas. La distinction entre cette logique et la logique féminine du pas-tout sera l'occasion de retracer les croyances sur la féminité dans la théorie psychanalytique et d'interroger les usages paradoxaux qui sont faits des positions dites féminine et masculine.

Mots clés: Féminité, hystérie, pas-tout, formules de la sexuation

\footnotetext{
*' Aix Marseille Université (Aix Marseille, França)
} 
Les rapports entre hystérie et féminité sont à jamais scellés par l'étymologie, qui épingle l'utérus migrateur - cette bête avide en mal de satisfaction - comme cause des désordres psychiques et corporels, jusqu'à en faire alors des dérèglements spécifiquement féminins. Le discours populaire persiste dans cette erreur - ces femmes qui jamais ne sauraient ce qu'elles veulent, à l'insatisfaction sans fond et à la plainte infinie, ces femmes qui en veulent toujours plus et qui ne savent dire ce qui, de leur manque, ne cesse de tant les agiter - perpétuant ainsi cette confusion qui consomme à l'excès l'identification de la position féminine avec la posture hystérique.

Qu'en est-il alors du discours psychanalytique? L'intrication hystérie et féminité est telle qu'aucun ouvrage psychanalytique portant sur l'une de ces questions ne manquera de faire référence à l'autre, alors même et tout l'intérêt des rapports entre hystérie et féminité loge en ce point - que sera systématiquement pointé, dans un mouvement apparemment contraire, le rapport d'exclusivité qu'elles entretiennent. Se décèle ainsi une ambivalence, révélatrice d'un paradoxe irréductible bien plutôt que d'une simple contradiction: intime liaison, en ce que l'hystérique est celle qui interroge au plus vif ce que c'est qu'être une femme; exclusivité, dans la mesure où 'devenir une femme et s'interroger sur ce qu'est une femme sont deux choses essentiellement différentes. (...) S'interroger est le contraire de le devenir" (Lacan, 1955-1956/1981, p. 200). Ambivalence seulement apparente, donc, qui n'est pas sans avoir trait au paradoxe même avec lequel l'hystérique tente de traiter l'énigme de la féminité.

La psychanalyse naît en se détournant du donner-à-voir de l'hystérique pour privilégier son dire, troquant ainsi le regard pour l'écoute, la place du maître pour une place qui n'est encore assurée d'aucune référence et que Freud cisèle à la mesure de ce qu'il en saisit. Il se laisse enseigner par ses premières patientes qui lui imposent de les laisser manœuvrer dans leurs associations - car ce sont bien des femmes qu'écoute d'abord Freud, des femmes malades d'une condition féminine qui ne se laisse pas réduire à ses aspects sociaux au biologiques. Nulle surprise alors à ce que la féminité se fasse objet de ses théorisations (Freud, 1931/1995a; 1932/1995b), théorisations connues pour avoir immédiatement suscité de houleuses controverses. 


\section{ARTIGOS}

Du côté lacanien, le célèbre aphorisme selon lequel "La femme n'existe pas", "la femme n'est pas-toute" (Lacan, 1972-1973/1975), témoigne d'une rupture franche avec les préceptes freudiens bien plutôt que du retour à Freud dont se réclame Lacan quant à son enseignement. Énoncés qui ont en leur temps — si ce n'est encore - excité la rage féministe, à ce que la femme soit encore et toujours parlée dans des termes négatifs ou privatifs. Rage qui se fait révélatrice d'une mécompréhension jusqu'au bout, en tant qu'elle se suspend bien plus à la formulation qu'à ce qu'elle implique - les termes choisis étant inquiétés de venir perpétuer le mouvement freudien de mutilation de la sexualité de la femme et de son désir, par l'insistance à user de la négation pour parler de la femme: "pas-toute", "n'existe pas".

Mais ce n'est bien évidemment pas sur le signifiant "femme" que Lacan fait porter une barre pour marquer son impossible écriture, mais sur le "La": " $L a$ femme, ça ne peut s'écrire qu'à barrer La. Il n'y a pas La femme, article défini pour désigner l'universel" (ibid., p. 68). Ea femme se fait ainsi l'inscription de ce qu'il n'existe pas de figure de "femme toute femme" qui permettrait, de par son statut d'exception, que les femmes forment un ensemble fermé, un universel féminin qui rendrait alors possible de dire un "toutes les femmes"; il n'y a pas dans l'inconscient de signifiant pour dire La femme, les femmes n'existent qu'à être prises une par une, dans une irréductible hétérogénéité. L'écriture de la barre sur le $L a$ a pour fonction de marquer ce défaut d'assise symbolique qui constitue la spécificité de la position féminine, à savoir qu'aucun signifiant spécifique ne peut garantir à une femme sa féminité ou assurer une identification propre à son sexe.

Ce $L a$ barré rompt sans conteste avec la croyance en une quelconque essence féminine qui résiderait dans une nature non-phallique - croyance soutenue par les opposants de Freud lors des violentes controverses qu'ont suscitées ses théorisations sur la féminité ${ }^{1}$ — sans pour autant suivre la voie freudienne qui s'est fourvoyée à réduire les enjeux féminins à une logique toute-phallique. Ni pas phallique, ni toute phallique, mais pas-toute phallique, telle est la proposition de Lacan, indissociable du La barré: pas-tout

\footnotetext{
${ }^{1}$ Il est traditionnellement admis de parler de ces controverses comme opposant les théorisations de Vienne à celles de Londres; et quoique cette seule nomination par les villes tende faussement à réduire cette querelle à l'expression de deux mouvements de pensée antagonistes entre eux mais intrinsèquement homogènes, il est toutefois possible de saisir l'enjeu de ces débats comme pouvant se formuler par l'alternative suivante: la femme est-elle "née ou faite"? (Lacan, 1960/1999b, p. 207) La féminité est-elle primaire ou secondaire?
} 


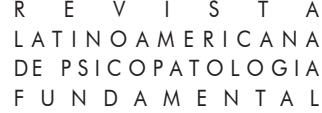

qui révèle à quel point il ne renvoie aucunement à un manque, mais bien à un supplément, puisqu'il signe que si toute jouissance, masculine comme féminine, s'inscrit dans le registre phallique, la jouissance féminine quant à elle se spécifie de n'être pas bornée par le phallus. Il y a un au-delà, une jouissance Autre, impossible à dire si ce n'est qu'elle peut s'éprouver, et dont l'amour mystique peut être l'une des manifestations. Car il s'agit encore de souligner que le pas-tout comme logique dite féminine ne permet pas de faire consister un ensemble limité et homogène, au même sens que le paradoxe de Russel selon lequel il n'y a pas "d'ensemble de tous les ensembles qui n'appartiennent pas à eux-mêmes" (Le Gaufey, 2006, p. 74). L'insistance de la négation pour parler du féminin n'a donc pas à être suspectée de mutiler la femme, dans la mesure où son usage a pour fonction de marquer que la femme ne peut se décliner qu'au singulier, un singulier particulier ne renvoyant à aucun universel.

$\mathrm{Au}$ regard de ces considérations, que signifie donc le paradoxe des rapports entre hystérie et féminité? Paradoxe à deux étages, puisqu'il concerne non seulement la façon bien particulière dont l'hystérique traite la barre qui affecte $L a$ femme - traitement qui nécessite effectivement

54 de distinguer la logique à l'œuvre dans l'hystérie de la logique féminine du pas-tout - mais également le double mouvement par lequel la psychanalyse saisit le lien entre hystérie et féminité, entre mise en équivalence et rupture radicale - au point alors d'exclure l'hystérie de la position féminine.

Cependant, et aussi pertinente que soit cette rupture de l'identité entre hystérie et féminité, ce mouvement ne témoigne-t-il pas d'un forçage au sein même de la théorisation psychanalytique quant à l'usage du "La femme n'existe pas", quand cet aphorisme est manié comme livrant les clés du devenir-femme, comme constituant un savoir sur la féminité "vraie"?

\section{Qu'est-ce qu'une femme?}

La question qu'est-ce qu'une femme? est épinglée par Lacan comme paradigmatique de l'hystérie et se fait l'emblème de toute l'incertitude qui existe à occuper une position féminine; ne pointe-t-elle pas en effet une absence, ou un trou, un impossible? Celui qu'il existerait une femme qui vaille comme modèle pour toutes les autres, une image de femme qui ait valeur d'idéal commun et qui, de son universalité, offrirait ainsi une direction à une identification proprement féminine. En quoi alors cette question 


\section{ARTIGOS}

constitue-t-elle non seulement une entrave, mais bien l'impasse dans laquelle s'égare et s'enlise l'hystérique quant au devenir-femme, alors même qu'elle ne semble que traduire et relayer le fait que $L a$ femme n'existe pas?

Si cette question dévoile la profonde incertitude sur ce que c'est qu'une femme, si elle témoigne en effet de l'absence d'une garantie symbolique à la féminité, l'insistance avec laquelle elle se met en jeu pour l'hystérique - au point même de constituer la mise de toutes les relations de désir ou d'amour - semble marquer, dans le mouvement contraire, à quel point cette question se fait appel passionné à un savoir sur $L a$ femme. Double mouvement révélateur du traitement qu'opère l'hystérique quant à $\mathrm{Ea}$ femme, dont la barre dans la version hystérique présentifie moins ce signifiant dans son inexistence que dans un simple statut d'absence - absence renvoyant alors à une présence possible, au fondement de la croyance sur laquelle se règle l'hystérique dans ses rapports à la féminité.

Si la logique hystérique se spécifie d'une récusation systématique quant à tout ce qui pourrait s'imposer comme un savoir définitif sur la femme (quitte à refuser les images de la féminité véhiculées par tel discours ou les places attribuées à la femme par tel autre, mais tout aussi bien à s'y identifier en tous points pour mieux les contester), cette récusation ne se soutient d'aucune conviction quant à ce qu'est La femme, mais seulement d'un questionnement ouvert à l'infini, dont on peut débusquer l'impulsion sans cesse renouvelée dans la croyance selon laquelle $t a$ femme (barré, donc) n'existe pas. Quelque chose de cette barre ne s'écrit pas sur l'universel féminin dans la syntaxe hystérique, n'affecte donc pas le "La" mais bien le "femme" dans un ratage perçu comme destin singulier, quand bien même serait-il commun pour toutes les femmes, comme une impuissance qui ressortit au registre de la castration et non pas au trou dans le symbolique.

Telle est l'erreur au cœur de la logique hystérique: l'absence de garantie symbolique à la féminité est saisie comme un dommage, un manque qu'il s'agirait alors de combler - lexique qui révèle à quel point la logique hystérique reste toute-phallique. Les rouages de l'hystérie sont à resituer dans la logique du tout ou rien propre au ravage (Lacan, 1972/2001, p. 465), qui trouve son origine dans la relation primaire à la mère et se perpétue dans les avatars de la relation amoureuse. Ce qui semble ici en jeu consiste en l'aboutissement d'une série d'équivalences: être ou pas le phallus pour la mère $=$ être ou pas le phallus pour l'homme aimé $=$ être ou pas La femme - simple continuation d'où peut être déchiffrée l'équivalence ultime entre La femme et le phallus. 
En d'autres termes, le “qu'est-ce qu'une femme?" marque que la solution mise en place par l'hystérique pour pallier le trou dans le symbolique consiste à aborder l'énigme de la féminité en la saturant par une question - question de savoir ce qu'est une femme en tant que cause du désir de l'Autre, substituant ainsi le phallus au manque d'un signifiant de La femme. Dire que l'hystérique ne croit pas en la barre portée sur le $L a$ ne revient pas à dire qu'elle croit en La femme, mais bien qu'elle traite la négation par une négation, sans que ne surgisse pour autant une quelconque positivation. Car l'idéal qu'elle substitue à l'absence du signifiant de La femme ne garantit en rien sa féminité, puisqu'il la laisse toute suspendue à la reconnaissance du désir de l'Autre, impulsant par-là la dynamique du désir, de l'amour et de la jouissance propre à l'hystérique, selon un modèle qualifié de masculin en tant qu'il se règle sur l'exception.

\section{La logique hystérique de l'exception}

Bien que ce soit l'absence d'une garantie symbolique à la féminité

56 qui impulse la logique hystérique, la solution qu'elle y trouve consiste en un recours exclusif à la logique phallique, du côté de l'être. Solution qui consiste à se faire objet cause du désir de l'Autre, dans la visée que quelque chose se dise d'un savoir sur ce qui, de la féminité, le fait ainsi désirer. Mais l'hystérique ne se veut pas n'importe quelle femme, elle ne se fait pas n'importe quel objet, dans la mesure où l'image de femme qu'elle incarne est soutenue de la croyance en une féminité idéalisée qui ne souffre pas d'être renvoyée au statut des autres femmes. Aussi, si la dérobade chère à l'hystérique se repère classiquement dans le refus opposé à la bascule d'objet de désir à celui d'objet de jouissance - l'hystérique s'offrant comme cause de désir mais s'esquivant comme objet de jouissance - ce refus ne tient finalement pas tant à cette bascule qu'à une autre: celle qui renverrait l'hystérique à l'interchangeabilité que confère le statut d'objet de désir. Tout se passe comme si le désir de l'autre - là même où il est littéralement appelé, invoqué - quand il en arrive à se manifester, ne la renvoyait toujours que trop à la classe indécise des femmes, du "comme toutes les femmes", la renvoyait à l'insoutenable d'une position qui ne concerne pas tant le refus d'être objet de désir — ni même objet de jouissance — que l'interchangeabilité qu'implique de fait cette place, ce risque aiguë de n'être qu'une, et non pas "la" Une. 


\section{ARTIGOS}

Être reconnue comme unique, être la seule, faire exception à la multitude des autres femmes, telles seraient autant de formules possibles pour énoncer la condition qui soutient le consentement hystérique, ce qui éclaire l'attente sur laquelle se règle son rapport au partenaire: que l'accès à un statut d'exception la marque d'un trait qui n'est pas tant trait commun que trait radicalement distinctif dans l'unicité qu'il est censé (re)marquer, en lieu et place du signifiant manquant. Il semble ainsi que l'hystérique ne condescende à être reconnue comme femme qu'à la condition, précisément, de se démarquer des autres femmes; à moins qu'il ne s'agisse plus exactement d'être remarquée - c'est à dire d'être marquée d'un trait autre, en plus, spécifique. Si l'hystérique en appelle au désir de l'autre pour reconnaître sa féminité, pour lui reconnaître ce trait qui la ferait femme, encore faut-il que ce signe qui lui est prêté l'extraie de la communauté des femmes, ou plutôt qu'il la constitue en tant que son exception - seule place où le désir de l'autre vaudrait pour garantie. Et c'est à cet endroit qu'émerge le revers de ce montage dans toute sa dimension de risque et d'impasse: car à ce que la reconnaissance de la féminité se retrouve toute suspendue au désir de l'autre, le rapport à l'autre peut aller jusqu'à friser le sacrificiel, et ne manquera jamais de relancer l'amère déception qui fait le lit de la plainte hystérique.

Aussi la position féminine se distingue-t-elle de la posture hystérique de tout l'écart qui existe entre une logique de la singularité et une logique de l'exception. Le devenir-femme comme création singulière trouve sa condition dans l'émergence de la féminité comme lieu vide ( $\mathrm{Ea})$, sans référence certes, mais ouvrant par là-même à la possibilité d'infinies inventions. Or, cette voie ne peut se retrouver qu'en rupture avec tout l'effort fourni par l'hystérique pour incarner l'exception féminine à tout prix. C'est dire quel poids de consistance l'universel féminin revêt pour l'hystérique, au point que la seule assurance qu'elle trouve à sa féminité consiste à faire objection aux "toutes les femmes", c'est à dire à être placée au-dehors de cette ligne d'équivalence insupportable. Et le ratage auquel ne manquera de conduire la solution hystérique se retrouvera sans cesse recouvert par la relance de l'idéal - non sans la laisser périodiquement en plan, désertée de sa croyance. Cette oscillation trahit la dimension ambivalente de cet idéal, car quand bien même ne parvient-il pas à fonctionner comme modèle ou comme garantie de féminité — dans la mesure où la circonscription de ses coordonnées demeure à chaque fois suspendue à au désir de l'autre et à sa reconnaissance — il n'en obture pas moins le trou dans le symbolique, en ce qu'il perdure à nourrir une attente du côté d'un savoir sur La femme. 


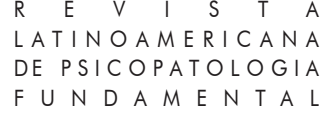

L'hystérique évolue ainsi dans la question de la féminité selon une logique toute phallique, bornée par une exception qu'elle se voue à incarner, à l'opposé de la logique du pas-tout qui consiste précisément, quant à elle, en l'objection à l'exception, objection qui relève d'un "traitement particulier de l'idéal. C'est un traitement par la destitution” (Pickmann, 2006, p. 56). Et c'est bien ce repérage qui fait situer l'hystérique du côté dit masculin; non pas parce qu'elle ferait l'homme au sens où elle se prendrait pour un homme - quoique cette option ne soit nullement inenvisageable - mais au sens où "l'hystérique ne veut rien savoir du pas-tout phallique" (Bruno, 2013, p. 287), et où fait l'homme tout sujet qui ordonne sa jouissance à l'horizon bouclé par une exception.

Que l'hystérique femme soit placée du côté homme montre suffisamment à quel point les formules lacaniennes dites masculines et féminines ne décalquent en rien l'anatomie, à quel point elles ne prétendent nullement à une lecture ontologique de la féminité et de la masculinité ; au contraire font-elles rupture d'avec toute croyance essentialiste en la différence des sexes de par la promotion d'une écriture logique situant la différence dans la façon dont la jouissance du sujet s'agence à la fonction phallique, en tout ou en pas-tout. Libre en effet au sujet de se placer du côté homme ou femme, 58 indépendamment de toute référence anatomique. ${ }^{2}$

\section{L'ambiguïté de "La femme n'existe pas"}

Est cependant décelable un certain usage de ces formules qui, malgré les dénégations, n'est pas sans retomber du côté de l'essentialisme dans la mesure où toute clinique féminine sera de fait référée au pas-tout - celui-ci en venant, d'une façon des plus contradictoires, à définir la position féminine, ce qui n'est pas sans laisser affleurer une croyance en un universel féminin. "Il y a une tendance à lier indéfectiblement les femmes et le Pas-tout qui repose sur une implicite évidence naturelle de ce lien. C'est une autre façon de reconduire une logique essentialiste, comme si, dès lors qu'on était biologiquement femme, il y avait nécessairement un accès à une jouissance Autre qui serait liée au Pas-tout phallique" (Blaquière, 2014, p. 75). Cette remarque conduit à envisager de façon critique tout modèle théorique proposant le pas-tout comme ce qui réglerait de fait le rapport des femmes à la castration ou, de

2 “On s'y range, en somme, par choix” (Lacan, 1972-1973/1975, p. 67). 


\section{ARTIGOS}

façon plus large, la construction de la féminité. Car si le pas-tout désigne la logique dite féminine par Lacan, l'inscription des femmes dans la fonction phallique ne semble pour autant pas systématiquement réglée sur ce mode-là. Lacan avait déjà souligné que la référence au sexe anatomique ne faisait pas figure de vecteur suffisant à inscrire le sujet d'un côté ou de l'autre du tableau de la sexuation. Certains auteurs continuent cependant à se repaître de cette énigmatique jouissance féminine dès qu'il s'agira de parler d'une femme, quand bien même cette jouissance Autre - Autre parce qu'"au-delà du phallus" (Lacan, 1972-1973/1975, p. 69) — ne semble pas ressortir dans le séminaire de Lacan comme constituant l'apanage de la condition féminine, mais bien celui de la position féminine. Malgré cette précision, quelque chose d'une inextricable confusion n'en règne pas moins, dans la mesure où l'ambition du séminaire Encore est clairement formulée comme tentative d'en dire plus sur la sexualité féminine, "de faire sortir du nouveau" (ibid., p. 54) du continent noir. Et la jouissance Autre référée alors à la jouissance autre que clitoridienne, "jouissance qu'on appelle comme on peut, l'autre justement, celle que je suis en train d'essayer de vous faire aborder par la voie logique (...) alors on l'appelle comme on peut, cette jouissance, vaginale" (ibid., p. 69).

Confusion des plans, donc? Et si tout psychanalyste se réclamant de l'enseignement de Lacan ne saurait ignorer que la condition anatomique ne dirige pas en tant que telle la sexuation, n'en persiste pas moins la tentation de s'engouffrer dans la brèche - et la jouissance Autre sera alors l'objet d'une traque systématique de la part des psychanalystes post-lacaniens quand il s'agit de la clinique des femmes: y a-t-il ou n'y a-t-il pas jouissance au-delà du phallus? Enquête semblant voiler une autre question: est-elle ou n'est-elle pas "vraie" femme?

La distinction hystérie-féminité semblerait en première analyse s'élever à l'encontre d'une telle confusion. Si ce n'est que la référence systématique de la femme au pas-tout se révèle bien plus subtile qu'il n'y paraîtrait au premier abord, en ce que ce systématisme ne se réduit pas à l'affirmation d'un lien incontestable de la femme à la jouissance Autre, mais qu'il est encore présent dans la seule présomption de ce lien, là-même où celui-ci est reconnu comme manquant. Or, à envisager ainsi l'hystérique comme une femme qui se fourvoierait quant à la construction de sa féminité, à concevoir la logique hystérique comme procédant d'une méprise, d'un refus, c'est dans cette dernière perspective que s'inscrit la distinction entre hystérie et féminité - faisant alors $\mathrm{du}$ "pas-tout" son horizon manquant et de la jouissance Autre l'inaccessible lieu de sa féminité échouée. Exclure l'hystérique de la position féminine évite certes 
le piège de la confusion entre sexuation et anatomie, mais au prix d'une autre confusion: celle de faire des formules de la position féminine, du "La femme n'existe pas", de la jouissance Autre et du pas-tout les clés de la féminité même, les coordonnées d'une féminité alors tenue pour "vraie".

\section{La vraie femme}

Les expressions "vraie femme" ou "vraie féminité" ne sont pas sans évoquer l'usage qu'en fait Lacan lui-même, à condition de noter que ces occurrences datent exclusivement de 1958, soit bien avant l'élaboration de "La femme n'existe pas". Ces formules se retrouvent dans le séminaire V (Lacan, 1957-1958/1998, p. 195), ${ }^{3}$ mais l'occurrence la plus connue (Lacan, 1958/1999a, p. 239) ${ }^{4}$ concerne l'identification opérée par Lacan entre l'acte de Madeleine détruisant une par une les lettres de Gide et l'acte de Médée tuant les enfants de Jason - rapprochement conduisant certains à situer la vraie femme "exactement à l'inverse de la mère" (Zafiropoulos, 2010, p. 227). Une femme dite "vraie", donc, d'être épurée de l'avoir phallique que représenterait la maternité - interprétation sujette à controverses, qui a néanmoins le mérite d'accentuer le geste de Lacan arrachant la féminité au seul destin maternel, impasse dans laquelle Freud avait confiné le devenir-femme.

Une autre lecture vise - sans souci d'anachronisme - à articuler les propos de Lacan de 1958 avec ses avancées ultérieures, voire à déceler dans l'affirmation d'une "vraie femme" les prémisses de la jouissance féminine dans son lien particulier au phallus; l'acte de Médée se retrouve alors hissé au rang de manifestation "pure" de la jouissance féminine, lecture qui trahit par-là la tentation de situer la femme, la "vraie", dans un régime au-delà du phallus se confondant alors tout à fait avec un régime non phallique - ce repérage faisant ainsi fi de la double flèche qui relie La femme au phallus et au $\mathrm{S}(\mathbb{A})$.

Aussi, faire perdurer toute référence à la "vraie femme" dans un registre lacanien après que la barre ait été portée sur le La semble n'être possible

\footnotetext{
3 "Cela vous indique aussi en quoi une féminité, une vraie féminité, a toujours un peu une dimension d'alibi. Les vraies femmes, ça a toujours quelque chose d'un peu égaré."

4 "Jusqu'où elle vint à devenir ce que Gide la fit être, reste impénétrable, mais le seul acte où elle nous montre clairement s'en séparer est celui d'une femme, d'une vraie femme, dans son entièreté de femme."
}

Rev. Latinoam. Psicopat. Fund., São Paulo, 20(1), 51-64, mar. 2017 


\section{ARTIGOS}

qu'au prix d'une incroyable torsion: celle de faire cohabiter la figure de la vraie femme (Médée par excellence) avec l'infigurable de Ła femme.

\section{Conclusion}

Au paradoxe avec lequel l'hystérique traite la barre sur La femme fait écho un paradoxe au sein même des théories psychanalytiques, lorsque l'avancée lacanienne selon laquelle "La femme n'existe pas" finit par fonctionner comme un obturateur, lorsque cette formule, reprise en ritournelle, finit, dans un revers des plus inattendus, par définir le devenir-femme.

La remise en cause de l'équivalence féminité-hystérie a non seulement le mérite de déjouer l'étymologie en distinguant les plans de l'anatomie et de la sexuation, mais également celui d'ouvrir un champ d'exploration de la féminité en dehors du seul paradigme hystérique. Si ce n'est qu'indiquer les seules coordonnées de la position féminine dans un au-delà de l'hystérie n'est pas sans laisser perplexe; la promotion d'une telle féminité épurée de toute hystérie ne vient-elle pas précisément reconduire la croyance hystérique selon laquelle il existerait une vraie femme? Existerait-il une femme dont le fonctionnement du désir et de l'amour pourrait se départir totalement de la logique de l'exception telle qu'elle a été décrite? Non pas, et c'est là que la subtilité du pas-tout reste à saisir, en ce que la jouissance qui lui est corrélée n'est nullement la négation ou le revers de la jouissance phallique, ne suppose pas une rupture de logique d'avec le réglage phallique, mais un supplément.

Ce n'est d'ailleurs pas à l'endroit de la jouissance Autre, dite "féminine", qu'a porté cette critique mais bien sur certains de ses usages, dits ici paradoxaux en ce qu'ils suturent formules de la sexuation féminine et coordonnées du devenir-femme, là où les tableaux de la sexuation, s'ils ont pour vocation d'"envisager de nouveau la question du féminin [...] non pas à partir de la question de l'emblème, de l'insigne, [...] de la mascarade; non pas à partir de la question des identifications, mais à partir de la question des jouissances" (Brousse, 2000), font advenir une ligne de faille inédite entre féminité et féminin, entre devenir-femme et position féminine.

Encore s'agirait-il de répéter que ces usages ne sont pas sans trouver leur ancrage dans une certaine ambiguïté présente dans les propos mêmes de Lacan. Et si l'on peut sans réserve affirmer que l'hystérique n'est pas une femme lacanienne, cela ne revient pas à dire qu'elle se méprend sur la féminité - au risque de ré-essentialiser cette dernière et d'en conclure alors que $L a$ femme reste singulièrement mal barré(e) au sein même de la théorie psychanalytique. 


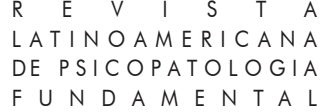

\section{References}

Blaquière, H. (2014). La jouissance négativement phallique. Figures de la psychanalyse, 27, 69-80.

Brousse, M-H. (2000). Qu'est-ce qu'une femme? Pont freudien. Repéré à $<$ http:// pontfreudien.org/content $/$ marie-h\% $\% 3 \% \mathrm{~A} 91 \% \mathrm{C} 3 \% \mathrm{~A} 8$ ne-brousse-quest-ce-quunefemme>

Bruno, P. (2013). Une psychanalyse: du rébus au rebut. Toulouse: Érès.

Freud, S. (1995a). De la sexualité féminine. In Euvres Complètes de Freud / Psychanalyse (t. XIX, pp. 8-28). Paris: PUF. (Original publié en 1931).

Freud, S. (1995b). La féminité. In Euvres Complètes de Freud / Psychanalyse (t. XIX, pp. 195-219). Paris: PUF. (Original publié en 1932).

Lacan, J. (1975). Le séminaire. Livre XX. Encore. Paris: Le Seuil. (Original publié en 1972-1973).

Lacan, J. (1981). Le séminaire. Livre III. Les Psychoses. Paris: Le Seuil. (Original publié en 1955-1956)

Lacan, J. (1998). Le séminaire. Livre V. Les formations de l'inconscient. Paris: Le Seuil. (Original publié en 1957-1958).

Lacan, J. (1999a). Jeunesse de Gide ou la lettre et le désir. In Écrits 2 (pp. 217-242). Paris: Le Seuil "Points Essais". (Original publié en 1958).

Lacan, J. (1999b). Propos directifs pour un Congrès sur la sexualité féminine. In Écrits 2 (pp. 203-214). Paris: Le Seuil "Points Essais". (Original publié en 1960).

Lacan, J. (2001). L'Étourdit. In Autres Écrits (pp. 449-495). Paris: Le Seuil. (Original publié en 1972).

Le Gaufey, G. (2006). Le pastout de Lacan. Paris: EPEL.

Pickmann, C-N. (2006). D'une féminité pastoute. La clinique lacanienne, 11, 43-63.

Zafiropoulos, M. (2010). La question féminine, de Freud à Lacan. Paris: PUF.

\section{Resumos}

(A mulher mal barrada. Crenças na mulher, da histeria às teorias psicanalíticas)

A pergunta "O que é uma mulher?" e a lógica da exceção pela qual a histérica se rege em sua relação com a feminilidade dão testemunho de sua crença de que não existe a barra colocada por Lacan sobre a Mulher. A distinção entre essa lógica e a lógica feminina do "não todo" será ocasião para situar novamente as crenças sobre 


\section{ARTIGOS}

a feminilidade no marco da teoria psicanalítica e para interrogar os usos paradoxais das posições "feminina" e "masculina".

Palavras-chave: Feminilidade, histeria, não todo, fórmulas da sexuação

(The not wholly barred Woman. Beliefs in Woman, from hysteria to psychoanalytic theories)

The question "What is a woman?" and the logic of exception on which a hysterical woman bases herself in her relationship with femininity reveal one of her beliefs: that the bar placed by Lacan on Woman does not exist. The distinction between this logic and the feminine logic of the "not-whole" is the opportunity to once again place beliefs on femininity within psychoanalytic theory and to question the paradoxical uses of "feminine" and "masculine" positions.

Keywords: Femininity; hysteria; not-whole; sexuation formulas

(La mujer mal barrada. Creencias en La mujer, de la histeria a las teorías psicoanalíticas)

La pregunta “¿Qué es una mujer?” y la lógica de la excepción, que rige a la histérica en su relación con la femineidad, atestiguan sobre su creencia de que no existe la barra colocada por Lacan sobre la mujer. La distinción entre esta lógica y la lógica femenina del "no todo" será la ocasión para volver a situar las creencias sobre la femineidad en la teoría psicoanalítica y para interrogar los usos paradójicos de las posiciones "femenina" $y$ "masculina".

Palabras clave: Femineidad, histeria, no-todo, fórmulas de la sexuación

(Die schlecht gestrichene Frau. Ansichten zur Frau, von der Hysterie zu den psychoanalytischen Theorien)

Die Frage „Was ist eine Frau?" und die Ausnahmelogik wonach sich die Hysterikerin in ihrem Bezug auf die Weiblichkeit richtet, zeugen von ihrem Glauben daran, dass der Strich, mit dem Lacan Die Frau gestrichen hat, nicht existiert. Die Unterscheidung zwischen dieser Logik und der weiblichen Logik des „nicht-alles “ ist Anlass dazu, die übernommenen Vorstellungen zur Weiblichkeit innerhalb der psychoanalytischen Theorie neu zu positionieren, sowie die paradoxe Benutzung ,weiblichen “ und ,, männlichen " Positionen zu hinterfragen.

Schlüsselwörter: Weiblichkeit, Hysterie, nicht-alles, Formeln der Sexuierung

（未被完全标签化的女人: 关于女人特质，从歇斯底里症到精神分析理论）

“女人是什么?” 的问题和建立在 例外逻辑 (lógica da exceção)之 上的歇斯底里症患者与女性特质的关系问题，是精神分析学家拉孔 (Lacan) 的女 性心理分析学说的基础, 阅读拉孔的著作, 我们发现拉孔并没有给女性特质加 


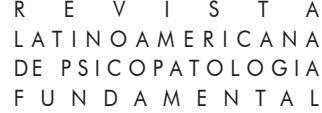

上任何标签。通过区分例外逻辑 (lógica da exceção) 和 “不都是” 的女性逻 辑 (lógica da feminilidade), 我们重新思考了精神分析理论中的女性特质问 题，并质疑从所谓的 “男性状态” 和 “女性状态” 中提取的二者悖论。 关键词：女性特性，歇斯底里，“不都是”，性化图式。

Citação/Citation: Fanny, C. (2017, março). La femme mal barrée. Croyances en La femme, de l'hystérie aux théories psychanalytiques. Revista Latinoamericana de Psicopatologia Fundamental, 20(1), 51-64.

Editores do artigo/Editors: Profa. Dra. Ana Maria Rudge e Profa. Dra. Sonia Leite

Recebido/Received: 25.8.2016 / 8.25.2015 Aceito/Accepted: 19.10.2016 / 10.19.2016

Copyright: (C) 2009 Associação Universitária de Pesquisa em Psicopatologia Fundamental/ University Association for Research in Fundamental Psychopathology. Este é um artigo de livre acesso, que permite uso irrestrito, distribuição e reprodução em qualquer meio, desde que $\mathrm{o}$ autor e a fonte sejam citados / This is an open-access article, which permits unrestricted use, distribution, and reproduction in any medium, provided the original authors and sources are credited.

Financiamento/Funding: A autora declara não ter sido apoiada ou financiada / The author has no support or funding to report.

Conflito de interesses/Conflict of interest: A autora declara que não há conflito de interesses / The author has no conflict of interest to declare.

\section{Fanny Chevalier}

Docteur en Psychologie clinique; Membre associée du Laboratoire de psychopathologie clinique: langage et subjectivité (LPCLS); Chargée de cours, Aix-Marseille Université (AMU); Psychologue clinicienne en CMPP.

Laboratoire de psychopathologie clinique: langage et subjectivité (LPCLS; EA 3278), Aix-Marseille Université (AMU),

29 av. Robert Schuman

13621 Aix-en-Provence cedex 1, France.

fanny.chevalier@neuf.fr

This is an open-access article, which permits unrestricted use, distribution,

(cc) BY-NC and reproduction in any medium for non-commercial purposes provided the original authors and sources are credited. 